ISASE 2020

\title{
Building a device-free system to prevent fractures in older people with musculoskeletal ambulatory disability symptom complex
}

\author{
Keiko Fukuroku*, Yugo Narita*, Hiroharu Kawanaka** \\ * Course of Nursing Science, Graduate School of Medicine, Mie University, 2-174 Edobashi, Tsu-shi, Mie 514-8507, Japan \\ **Graduate School of Engineering, Mie University, 1577 Kurima-machiya, Tsu-shi, Mie 514-8507, Japan
}

\begin{abstract}
This study aimed to measure the physical ability to cope with daily life and perceptions of older people at high risk of sarcopenia. We surveyed parameters to predict the risk of sarcopenia and factors worsening the condition. We used a pretest among five participants to check the relevance and validation of measures for (1) physical function, (2) physical activity, (3) environmental factors, and (4) subjective daily living activities. We plan to use these as the basis to develop a device-free sensor system to predict the onset and deterioration of sarcopenia in older people. Four participants had reduced muscle mass or left and right imbalances in one or both legs. All participants had consistent levels of vigorous and moderate activity and good sleep status. They also all kept to the protocol and wore the equipment for a whole month without any missing data. We will use the results of this pilot survey to identify changes and influencing factors for sarcopenia among older people.

Keywords: Sarcopenia, Fracture prevention, Musculoskeletal ambulatory disability symptom complex (MADS), Older people, Device-free system
\end{abstract}

\section{INTRODUCTION}

In an aging society, sarcopenia is a global health issue. Its prevalence is about $20-80 \%$ among those aged 70 years or older globally. In total, 2.7 million people in Japan are estimated to have this condition, and the number is expected to increase rapidly in the future [1]. However, the pathological mechanism of sarcopenia is not clear, and there are few studies with a high level of epidemiological evidence. Extending healthy life expectancy by preventing sarcopenia is a key issue in an aging society, especially from a cost-effectiveness point of view.

We have previously implemented low-load, long-term exercise interventions for musculoskeletal ambulatory disability symptom complex (MADS) in older patients and reported maintenance of physical function and improvement of ability to perform daily activities [2]. It is important to consider individual support for older people, because the degree of pain and fatigue influences physical activity in older people [3]. It is therefore necessary to capture changes in feelings or emotions using an objective tool, as well as using physical data such as amount of muscle mass, grip, or $10 \mathrm{~m}$ walking speed, behavioral data (for example, activity strength, walking distance, and acceleration of posture change) and environmental data (such as weather).

However, very few longitudinal studies have reported on a range of diverse and complex influencing factors, such as psychological state [4,5] and climate [6]. The technology for observing daily activities using sensors in monitoring systems has advanced, and it is now possible to collect and analyze large amounts of data. However, the costs and physical and mental burdens associated with wearing sensor equipment have led to difficulties in its continuous use. There is considerable demand for a system imposing a relatively small burden, requiring careful selection of monitoring items, and device-free. It is essential to clarify factors such as when, where and what data should be monitored and what criteria should be used to provide basic information for the prediction of onset and deterioration of sarcopenia and fracture prevention in older people with MADS.

Careful selection of monitoring items, supported by the development of cheaper and smaller sensing devices, should improve the technology for monitoring daily activity without creating a burden for those involved.

\section{OBJECTIVE}

We carried out a pretest to assess the relevance and validation of measures of (1) physical function, (2) physical activity, (3) environmental factors, and (4) subjective activities of daily living, and used this as the basis for developing a device-free sensor system to predict the onset and deterioration of sarcopenia in older people with MADS. 


\section{METHOD}

\subsection{Study design}

The study will eventually be longitudinal, over one year, but the pretest measurement period was set at one month.

\subsection{Participants}

We recruited five participants aged over 65 years with MADS, all of whom attended the monthly health class for local residents at the hospital.

\subsection{Measures and devices}

We used a questionnaire survey and took physical measurements. We also measured physical activity, environmental factors and subjective daily living conditions.

The MotionWatch 8 is a triaxial actigraphy watch from CamNtech, inventors of the Actiwatch [7]. It can be used to record daytime physical activity and report on levels of moderate to vigorous physical activity and sleep. Participants wore the watch on their wrist continuously for one month. Actual sleep time was expressed as a percentage of assumed sleep time. Sleep efficiency is actual sleep time expressed as a percentage of time spent in bed. Immobile time is given as a percentage of assumed sleep time. The Fragmentation Index shows the degree of fragmentation of the sleep period, and can be used as an indication of the sleep quality (or the lack of it).

The environmental sensor used was from OMRON. It was an ultra-compact multifunction sensing component, measuring temperature, humidity, barometric pressure, light intensity, sound, acceleration, VOC, and other environmental information, and has a wireless communication function [8]. Participants had them installed in the room where they spent most time at home, and they were in place for one month.

A self-completed activity $\log$ was used to check subjective daily living conditions for seven days.

Subjective pain in areas of the body was collected using a questionnaire with visual analogue scales (VAS). The score ranges from 0 (free of pain) to 100 (extreme pain).

\subsection{Ethical approval}

This study was approved by the Research Ethics Committee of the Faculty of Medicine, Mie University (No. U2019-028, November 2019).

\subsection{Data analysis}

Data analysis of descriptive statistics used a statistical application, SPSS Ver.22.0.

\section{RESULTS}

The study participants included four women and one man. Table 1 shows their demographic information. Their average age was $74.8 \pm 4.2$ years. The average pain value was $43.0 \pm 17.4$ and the most painful areas were shoulder, hip, knee and ankle. For questions such as "Do you like activities?", and "Do you keep up your activities?", all participants chose either "very" or "slightly". Two participants met the criteria for sarcopenia in the skeletal muscle index, However, not all of them were classified as having sarcopenia based on other judgment items such as walking speed and grip strength.

Table 1. Demographic information of the participants

\begin{tabular}{ccccccc}
\hline NO & age & sex & BMI & SMI $\left(\mathrm{kg} / \mathrm{m}^{2}\right)$ & grip $(\mathrm{kg})$ & $\begin{array}{c}5 \mathrm{~m} \\
\text { walking } \\
\text { speed }(\mathrm{m} / \mathrm{s})\end{array}$ \\
\hline 1 & 73 & F & 31.2 & 7.0 & 21.5 & 1.6 \\
2 & 78 & F & 31.1 & 6.6 & 32.3 & 1.3 \\
3 & 74 & F & 23.6 & $5.7 \downarrow$ & 18.9 & 1.2 \\
4 & 74 & F & 24.3 & 6.4 & 29.9 & 1.1 \\
5 & 83 & M & 23.7 & $6.8 \downarrow$ & 34.8 & 1.4 \\
\hline Mean \pm SD & $74.8 \pm 4.2$ & & $26.8 \pm 4.0$ & $6.5 \pm 0.5$ & $27.5 \pm 6.9$ & $1.3 \pm 0.2$ \\
\hline
\end{tabular}

Table 2 shows the degree of muscle development (segmental lean analysis) for each part of the body, including right arm, left arm, trunk, right leg, and left leg, using Inbody. Four participants had reduced muscle mass or left and right imbalances in one or both legs.

Table 2. Segmental lean analysis

\begin{tabular}{cccccc}
\hline NO & $\begin{array}{c}\text { LBM of } \\
\text { Right } \\
\text { Arm(kg) }\end{array}$ & $\begin{array}{c}\text { LBM of } \\
\text { Left } \\
\text { Arm(kg) }\end{array}$ & $\begin{array}{c}\text { LBM of } \\
\text { Trunk(kg) }\end{array}$ & $\begin{array}{c}\text { LBM of } \\
\text { Right } \\
\text { Leg(kg) }\end{array}$ & $\begin{array}{c}\text { LBM of } \\
\text { Left Leg(kg) }\end{array}$ \\
\hline 1 & 2.2 & 2.2 & 18.6 & $5.6 \downarrow$ & $5.6 \downarrow$ \\
2 & 1.8 & 1.7 & 16.2 & $5.4 \downarrow$ & $5.3 \downarrow$ \\
3 & 1.6 & 1.5 & 15.2 & 5.2 & $4.8 \downarrow$ \\
4 & 2.0 & 2.0 & 17.8 & 5.9 & 5.8 \\
5 & 2.4 & 2.3 & 20.0 & $6.7 \downarrow$ & $6.9 \downarrow$ \\
\hline Mean \pm SD & $2.0 \pm 0.3$ & $1.9 \pm 0.3$ & $17.6 \pm 1.9$ & $5.7 \pm 0.6$ & $5.7 \pm 0.8$ \\
\hline
\end{tabular}

Table 3 shows the activity levels measured by the MotionWatch. Activity intensity was classified into vigorous, moderate, low, and sedentary. All participants had very little vigorous activity and their level of moderate activity averaged 2:24:46 hours per day. The daily record showed that four participants went out every day, and the fifth went out on five days of the week (Table 4). 
Table 3. Activity level

\begin{tabular}{ccccc}
\hline NO & total Vig & total Mod & total Low & total Sed \\
\hline 1 & $0: 47: 53$ & $3: 01: 41$ & $7: 10: 26$ & $12: 59: 59$ \\
2 & $0: 21: 23$ & $2: 10: 58$ & $8: 05: 51$ & $13: 21: 48$ \\
3 & $0: 19: 50$ & $2: 19: 07$ & $9: 17: 00$ & $12: 04: 03$ \\
4 & $0: 43: 48$ & $2: 54: 57$ & $7: 21: 38$ & $12: 59: 37$ \\
5 & $0: 26: 58$ & $1: 37: 10$ & $7: 10: 30$ & $14: 45: 22$ \\
\hline Mean & $0: 31: 58$ & $2: 24: 46$ & $7: 49: 05$ & $13: 14: 09$ \\
\hline \multicolumn{4}{c}{ time(hh:mm:ss) }
\end{tabular}

Table 4. Daily activity record

\begin{tabular}{ccccc}
\hline No & $\begin{array}{c}\text { leave-out } \\
\text { time/week }\end{array}$ & $\begin{array}{c}\text { leave-out } \\
\text { time/day(range) }\end{array}$ & $\begin{array}{c}\text { indoor } \\
\text { time/week }\end{array}$ & $\begin{array}{c}\text { indoor } \\
\text { time/day(range) }\end{array}$ \\
\hline 1 & 41.5 & $3.0-10.5$ & 37.5 & $1.0-10.5$ \\
2 & 15.5 & $0-3.5$ & 69.5 & $7.0-12.5$ \\
3 & 20.5 & $1.5-7.0$ & 54.5 & $3.0-11.0$ \\
4 & 30.5 & $1.0-7.0$ & 29.5 & $1.5-7.5$ \\
5 & 19.0 & $1.0-5.5$ & 55.0 & $5.5-10.0$ \\
\hline Mean \pm SD & $25.4 \pm 10.6$ & & $49.2 \pm 15.8$ & \\
\hline
\end{tabular}

Table 5 shows the sleep analysis. Overall, the participants' sleep status was good.

\section{Table 5. Sleep analysis}

\begin{tabular}{ccccc}
\hline No & $\begin{array}{c}\text { actual sleep } \\
(\%)\end{array}$ & $\begin{array}{c}\text { sleep } \\
\text { efficiency }(\%)\end{array}$ & $\begin{array}{c}\text { immobile time } \\
(\%)\end{array}$ & $\begin{array}{c}\text { Fragrmentation } \\
\text { index }\end{array}$ \\
\hline 1 & 89.1 & 86.4 & 92.2 & 34.2 \\
2 & 86.1 & 84.2 & 88.5 & 35.6 \\
3 & 93.1 & 89.8 & 94.2 & 26.0 \\
4 & 89.6 & 86.1 & 91.9 & 30.8 \\
5 & 84.2 & 76.7 & 86.5 & 52.4 \\
\hline Mean \pm SD & $88.4 \pm 3.4$ & $84.6 \pm 4.9$ & $90.7 \pm 3.1$ & $35.8 \pm 10.0$ \\
\hline
\end{tabular}

\section{DISCUSSION}

This study asked five participants to wear a motion watch for one month, as a pretest for a longer study. Participants in studies of this type often forget to wear the device, resulting in data loss, and making it difficult to obtain data for all days and periods [9].
We asked participants to wear a waterproof watch for 24 hours a day, every day. There were no missing data in this study because all participants wore the equipment at all times. A detailed record was also obtained for one week This suggests that future longitudinal research may be able to obtain data from a large number of participants with high adherence. We used the watch to obtain and analyze data on both activity and sleep. However, accurate activity analysis requires a lumbar attachment. This means that obtaining accurate data of several types requires multiple devices, which places a burden on participants. Behavior recognition research using advanced technology is often based on wearable devices with cameras, acceleration sensors, microphones, and the like mounted on the wrist. Research and development of equipment that can accurately measure the intensity of a wide variety of activities is progressing [10]. In recent years, research has used device-free behavior recognition, for example, with a radio wave transceiver such as Wi-Fi installed in the environment, actions are recognized from signal strength and the signal propagation path, which changes with human movement [11]. However, these methods are costly to introduce, require maintenance and can be hard to use continuously. It is therefore important to find a system that provides low-cost, casual daily action sensing [12]. We think that it is possible to develop a device-free sensor system by incorporating an engineering perspective and promoting collaboration between nursing and engineering (see Fig. 1). Our future work aims to acquire and analyze subjective and objective data from older people with MADS on a longitudinal basis and over a long period. We also plan to determine monitoring items that provide useful information on trends in gait and stability, especially decreases.

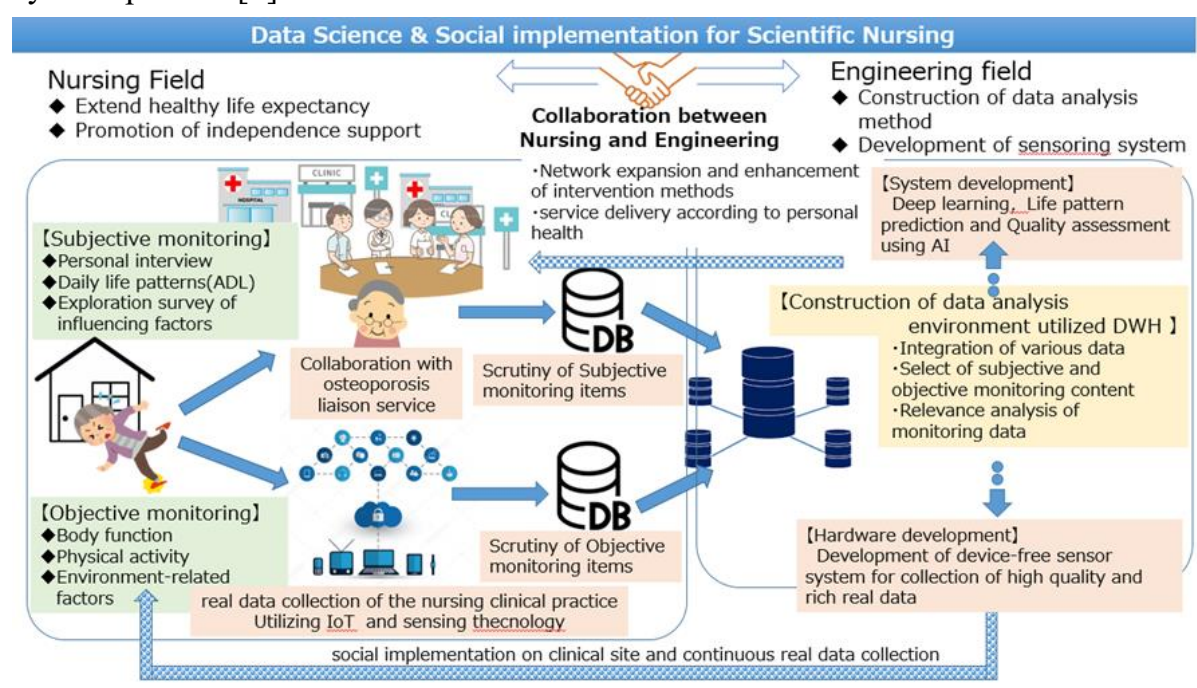

Figure 1 Data science and social implementation for scientific nursing 
Our research will also consider changes in daily life movements and life patterns of older people with MADS, and the factors that influence these to greater and lesser degrees. This should enable us to identify factors that influence the progress of MADS in older people, and select aspects to monitor. By promoting the development and practical application of device-free systems through collaboration between nursing and engineering, we can make data collection simpler, and provide high-quality, abundant real-life data. We want to construct a nursing data analysis environment using a data warehouse, and integrate various data obtained from large-scale prospective cohort studies to assess their relevance. The results will be used to promote effective independence support and establish and spread global standardized intervention methods.

\section{ACKNOWLEDGMENTS}

This study was conducted as part of a project supported by the Scientific Study Furtherance Fund grant, Japan Society for Promotion of Science, No. 19K10850 and OKASAN-KATO FOUNDATION. Title: Basic research on building a device-free system for fracture prevention among older people with musculoskeletal ambulatory disability symptom complex (leader, Keiko Fukuroku). We thank Melissa Leffler, MBA, from Edanz Group (https://en-author-services.edanzgroup.com/) for editing a draft of this manuscript.

\section{REFERENCES}

1. Center for Gerontology and Social Science, sarcopenia practice guidline 2017 (in Japanese) http://www.ncgg.go.jp/cgss/news/20180117.htm (accessed on $20^{\text {th }}$ January, 2020)

2. Fukuroku K, The intervention of fall prevention exercise: physical fitness transition for 1year and follow-up survey after the intervention. J Japan Society of Nursing Research. 34(3) pp328, 2011. (in Japanese)

3. Saito T, Sakata M, Kumagai S.: Physical activity epidemiology of chronic musculoskeletal pain in elderly adults, Journal of Health Science, Kyusyu University, 39,19-39,2017. (in Japanese)

4. Tsunoda K, Okura T: Psychological status, social networks, and physical activity in older adults, Japan journal of test and evaluation of physical education and sports, 11, 1-11, 2011. (in Japanese)

5. L Burton, S Shapiro, P German.: Determinants of Physical Activity Initiation and Maintenance among Community-Dwelling Older Persons, Preventive Medicine, 29(5), 422-430, 1999.

6. Aoyagi Y: The Nakanojo Study: an interdisciplinary study in the habitual physical activity and health of elderly people. IGAKUNOAYUMI, 523(9), 793-798, 2015. (in Japanese)

7.https://www.camntech.com/products/motionwatch/ motionwatch-8-overview

(accessed on 20th January, 2020)

8.https://www.omron.co.jp/ecb/productinfo/sensor/iot-sensor/environmental-sensor (accessed on 20th January, 2020)

9. Hara T, Matsumura Y, Yamamoto M. et al: The relationship between body weight reduction and intensity of daily physical activities assessed with 3-dimension accelerometer: Jpn. J. Phys. Fitness Sports Med, 55, pp.385-392, 2006. (in Japanese)

10. Sasai H: Assessing sedentary behavior using wearable devices: An overviewand future directions. J Phys Fitness Sports Med, 6(3), 135143, 2017. (in Japanese)

11. W Wang, J Liu, Y Chen. et al: E-eyes: device-free location-oriented activity identification using finegrained WiFi signatures. Mobi Com, 2014, 617-628, 2014.

12. Ohara K, Maekawa T, Kishino Y. et al: Transferring positioning model for device-free passive positioning based on Wi-Fi signals. Information Processing Society of Japan. 57(2), 406-415, 2016. (in Japanese) 